\title{
OPEN Genomic analysis of WD40 protein family in the mango reveals a TTG1 protein enhances root growth and abiotic tolerance in Arabidopsis
}

\begin{abstract}
Lin $\operatorname{Tan}^{1}$, Haron Salih ${ }^{1,2 \bowtie}$, Nwe Ni Win Htet ${ }^{1,3}$, Farrukh Azeem ${ }^{1}$ \& Rulin Zhan ${ }^{1 \bowtie}$
WD40 domain-containing proteins constitute one of the most abundant protein families in all higher plants and play vital roles in the regulation of plant growth and developmental processes. To date, WD40 protein members have been identified in several plant species, but no report is available on the WD40 protein family in mango (Mangifera indica L.). In this study, a total of 315 WD40 protein members were identified in mango and further divided into 11 subgroups according to the phylogenetic tree. Here, we reported mango TRANSPARENT TESTA GLABRA 1 (MiTTG1) protein as a novel factor that functions in the regulation of Arabidopsis root growth and development. Bimolecular fluorescence complementation (BiFC) assay in tobacco leaves revealed that MiTTG1 protein physically interacts with MiMYBO, MiTT8 and MibHLH1, implying the formation of a new ternary regulatory complex (MYB-bHLH-WD40) in mango. Furthermore, the MiTTG1 transgenic lines were more adapted to abiotic stresses (mannitol, salt and drought stress) in terms of promoted root hairs and root lengths. Together, our findings indicated that MiTTG1 functions as a novel factor to modulate proteinprotein interactions and enhance the plants abilities to adjust different abiotic stress responses.
\end{abstract}

WD40 repeat domains (WD40 domain-containing proteins) play important roles in diverse biological functions such as cytokinesis, cell division, apoptosis, meristem organization, floral development, cytoskeleton assembly, protein trafficking, chromatin modification, and gene transcriptional mechanism ${ }^{1-3}$. WD40 proteins are characterized as core residues of certain conserved motifs (44-60 amino acid) with glycine-histidine (GH) at the N-terminus and tryptophan-aspartic acid (WD) pair at the $\mathrm{C}$ terminus ${ }^{4}$. The name of WD40 proteins originate from the conserved WD regions and the core residue length of a single repeat contains about 40-60 amino-acids ${ }^{5,6}$. WD40 proteins are highly prevalent in all eukaryotes and rarely found in prokaryotic organisms ${ }^{7}$. WD40 protein motifs fold into seven-bladed $\beta$-propeller domain repeats which act as a scaffold for various protein-protein interactions and form functional complexes ${ }^{1}$. Generally, the functions of WD40 proteins need to be assisted in protein-protein interactions, which have no intrinsic enzymatic activities ${ }^{8}$. The interaction between WD40 repeat proteins, MYB and bHLH transcription factors have been widely investigated in flavonoid biosynthesis ${ }^{9}$. Also, the TTG1-bHLH-MYB complex is directly involved in the regulation of root growth and trichome patterning on the Arabidopsis leaf ${ }^{10}$. Several WD40 repeat proteins are associated with epidermal traits in Arabidopsis, Perilla and maize ${ }^{11}$. Recently, in Arabidopsis GIGANTUS1 is connected with biomass accumulation and seed germination through a protein-protein interaction ${ }^{7}$. WD40-REPEAT 5a plays an important role in drought tolerance through the regulation of nitric oxide accumulation ${ }^{12}$. Another WD40 protein (XIW1) is directly involved in the association of the stability ABI5 and ABA responses in Arabidopsis ${ }^{13}$. TaWD40D is significantly associated with wheat responses to abiotic stress ${ }^{14}$. Highlighting the features of WD40, its significance and relevance in the study of mangoes have to be elaborated. Previously, the genome-wide identification of the WD40 protein family has been carried out in Arabidopsis ${ }^{4}$, cotton $^{15}$, foxtail millet ${ }^{2}$, rice ${ }^{16}$, wheat ${ }^{17}$, human ${ }^{18}$ and peach ${ }^{19}$. Whereas, no study has been performed to identify these proteins in mango (Mangifera indica L.), which is one of the most important tropical fruit with high economic value worldwide. Mango belongs to the Anacardiaceae family, also known as the "king of fruits", and is a fruit with great dietary value ${ }^{20-22}$. The nutrient supply of mango includes calories, vitamins, minerals and fiber ${ }^{23}$. Agro-industrial residues and the flesh of mango have

\footnotetext{
${ }^{1}$ Hainan Key Laboratory of Banana Genetic Improvement, Haikou Experimental Station, Chinese Academy of Tropical Agricultural Sciences (CATAS), Haikou 571101, Hainan, China. ${ }^{2}$ Crop Sciences, Faculty of Agriculture, Zalingei University, Central Darfur, Sudan. ${ }^{3}$ Microbiology Laboratory, Biotechnology Research Department, Kyaukse 05151, Myanmar. ${ }^{\circledR}$ email: salih234@yahoo.com; zhanrulin555@163.com
} 
some bioactive compounds, containing nutrient and non-nutrient elements with biological properties that act mostly through redox mechanisms ${ }^{20}$. Each mango fruit breed varies in shape, color, size, taste, flavor and fiber content $^{24}$. The exocarp part grows into a leathery protective skin which is smooth, waxy and green, containing lenticels that emerged from stomata ${ }^{22}$. Based on cultivars, once mango is ripe, the fruit skin changes to a yellow sparsely marked with red or pale green ${ }^{23}$. In this study, we identified 315 WD40 protein members in mango at a genomic analysis level, including their domain numbers, evolutionary relationship, gene location and predicted molecular functions. Besides, we reported the molecular cloning of cDNA encoding a mango WD40 protein, MiTTG1 (Mi01g20920), an ortholog of the Arabidopsis AT5G24520 (TTG1) which promotes root growth and enhances osmotic, salt and drought stress tolerance in Arabidopsis. This work will provide a solid platform to better understand the functional roles of MiTTG1 gene and will help further investigation on the biological function and molecular mechanisms of these proteins family in mango.

\section{Results and discussion}

Identification, sequence characterization, subcellular localization prediction of the mango fruit WD40 repeat proteins. In order to identify the members of the WD40 protein family in the mongo genome database ${ }^{25}$, blast profiles (HMM research and local blast) were used as a query within the mango genome project. We identified 485 protein sequences in the mango genome database. After removing redundant sequences based on the Perl program, WD40 candidate protein sequences were investigated manually with the SMART online and Pfam database for the presence of the WD40 domain (Supplementary Table 1). Finally, a total of 315 protein members were identified in the mango genome database, which varied from 1 to 12 repeat domains (Supplementary Table 1). However, only a single copy of WD40 protein, MiTTG1 (four repeat domains) was identified to be the ortholog of Arabidopsis TTG1 in the mango genome database. In recent years, several WD40 proteins that are orthologs to the Arabidopsis TTG1 gene have been identified in some plant species, such as cotton (Gossypium hirsutum), Prunus persica, Punica granatum and maize (Zea mays) ${ }^{26-29}$. In studied plant species, It was found that there was only one copy of gene-encoding WD40 repeat protein (TTG1) ${ }^{26-29}$. Besides the divergence of conserved WD40 repeat domains, the protein encoded by mango WD40 gene family were significantly varied in the numbers of amino acids, physicochemical properties and divergence in the subcellular localization within a cell (Supplementary Table 1). The amino acid sequences of the 315 mango WD40 protein sequences varied in lengths from 84 to 3604 amino acids, with an average length of 655.4 amino acids (Supplementary Table 1). To explain the potential functions of the proteins encoded by the WD40 gene family under investigation, understanding their physiochemical features are vital, for example, the proteins can be divided depending on their molecular mass (size) and isoelectric point (charge) properties and their abundance then determined subsequently ${ }^{30}$. The molecular weights were largely varied between 9644.89 and 400,731.34 Da (Dalton) with an average of 72,516.59 Da. The investigation of the subcellular localization of 315 mango WD40 protein members was predicted by WoLF PSORT online tool. The result revealed that 166 of WD40 proteins were localized in the nucleus, 78 in chloroplasts and 43 in the cytosol while the remaining 28 WD40 proteins were localized in various subcellular membranes, such as mitochondria, cytoskeleton, peroxisome and plastid (Supplementary Table 1). This finding implies that WD40 proteins might be involved in regulating various physiological features of plant growth and developmental processes under different environmental conditions. The silico mapping of MiWD40s on chromosomes showed an uneven distribution of the genes on all the 20 chromosomes of mango. Three hundred and four (304) WD40 genes were distributed across 20 chromosomes in the mango genome while 11 genes were mapped to the scaffold (unknown chromosome). Some mango chromosomes and chromosomal positions had a high density of WD40 gene members while others do not (Supplementary Fig. 1). The highest densities of WD40 genes were detected on chromosome 3 with 34 genes, and the lowest densities of WD40 genes were showed on chromosomes 13 and 15 with 7 genes for each.

Phylogenetic tree and conserved motif analysis, GO annotation of the mango WD40 proteins family. The evolutionary relationship analysis of the mango WD40 protein family was performed by the construction of an un-rooted phylogenetic tree using the NJ (neighbor-joining) method, with 1000 bootstrap replicates. According to the protein sequence similarities, the 315 mango and 107 Arabidopsis WD40 protein members were divided into 11 subgroups, which varied in number from 16 to 75 protein members (Fig. 1A). The bootstrap value for several subgroups of the neighbor-joining tree was not high as a result of reasonably

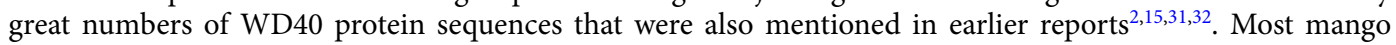
WD40 proteins showed high similarities within subgroups of phylogenetic tree analysis. This result was in agreement with previous reports in cotton and foxtail millet ${ }^{2,15}$. WD40 proteins family from mango and Arabidopsis are consistently present at intervals around the phylogenetic tree analysis. Specifically, based on the protein sequence similarities, each mangoWD40 repeat protein has a matching homolog in Arabidopsis thaliana. This result is consistent with the previous findings which showed that the majority of Arabidopsis WD40 proteins have a corresponding homolog WD40 protein in the plant kingdom ${ }^{4}$. Further investigation was done to predict the multiplicity of conserved motifs within $315 \mathrm{MiWD} 40$ proteins using the MEME program. This analysis identified ten conserved motifs that were labeled as motif 1 to motif 10 (Supplementary Fig. 2). The result revealed that motif 1 is the most conserved motifs among the ten motifs, being present in all mangoWD40 proteins. Motifs 2 and 4 were in the second and third most abundant motifs, respectively (Supplementary Fig. 2). Most WD40 proteins within the same subgroups had a common motif in terms of motif distribution and composition, which indicated the WD40 protein members within a given subgroup, may have similar functional roles. Furthermore, a certain unique conserved motif was detected in a particular subgroup, which revealed a stronger sign of involvement of this motif in specific roles within the higher plants. The gene ontology (GO) annotation was conducted using the OmicsBox/Blast2Go (https://www.blast2go.com/) tool, and it predicted the potential 

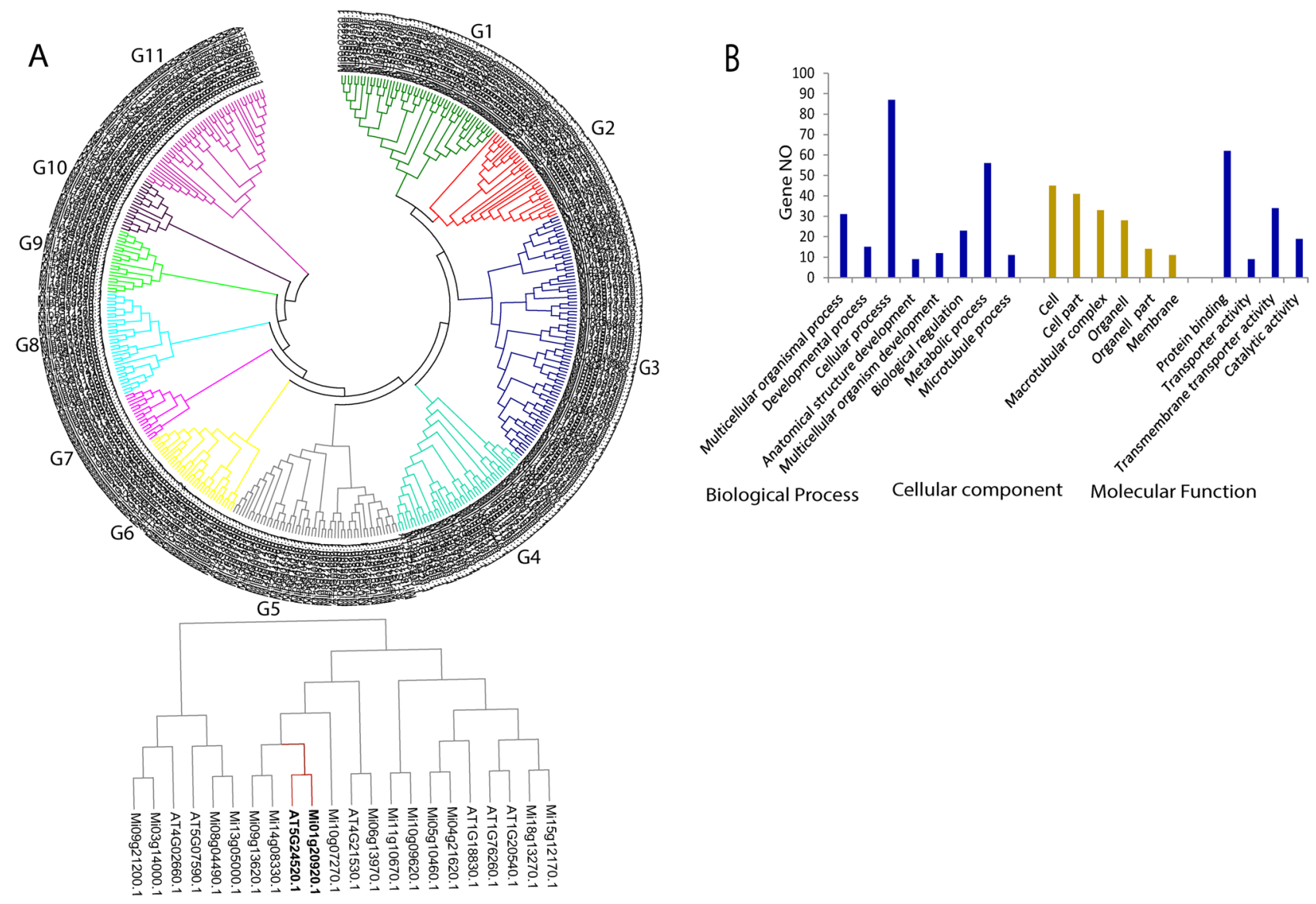

Figure 1. Functional analysis of mango WD40 proteins family. (A) Phylogenetic tree relationship between mango and Arabidopsis of WD40 proteins was conducted by MEGA 6.0 using the Neighbor-Joining (NJ) method. The11 subgroups are shown in colors. (B) GO annotation analyses of mango WD40 proteins were examined for their functions in biological process, molecular functions and cellular components.

function of WD40 proteins. Mango WD40 proteins were categorized into three groups by GO analysis based on their involvement in biological process, cellular component and molecular function. Accordingly, the WD40 proteins were categorized into 8 groups of biological processes; a significant number of WD40 proteins were associated with the cellular process, metabolic process, multicellular organismal process, biological regulation and developmental process (Fig. 1B). In terms of the cellular component prediction of WD40 proteins, they were relatively enriched in the cell, cell part, macrotubular complex, organelle, organelle part and membrane. In molecular functions, the WD40 proteins were found to be associated with protein binding, transmembrane transporter activity, catalytic activity and transporter activity (Fig. 1B) which possibly support their involvement in protein-protein interaction networks. In the previous finding, it was noticed that some members of WD40 proteins physically interact with bHLH and MYB transcription factors, which are involved in different aspects of plant growth and development ${ }^{33-37}$.

MiTTG1 encoded WD40-repeat protein. The mangoTTG1gene (MiTTG1), Mi01g20920 (ortholog of AT5G24520) possessed a complete ORF of 1014 bp encoding a protein sequence containing 337 amino acids with a theoretical pI and molecular mass of 8.66 and 67,306.6 Da, respectively (Supplementary Table 1). There were four WD-repeat domains in MiTTG1 at 66 107, $112 \sim 157,160 \sim 198$, and 249 289 amino acids (Fig. 2A). The knowledge about the protein interaction network of the target protein is a key factor in spurring the investigation of the protein involvement in plant growth and developmental processes such as signal transduction, cell formation, pattern establishment, organ development, and plant defense ${ }^{8,38,39}$. To understand protein interaction between TTG1 gene and other proteins in plants, the STRING database (https://string-db.org/) was used to perform the computational analysis of protein-protein interactions (Fig. 2B). The result of functional protein association networks of the mango TTG1 gene showed that this protein can physically interact with the 4 bHLH proteins (TT8, GL3, EGL3 and ATMYC1), 3 MYB proteins (MYB0, TT2 and CPC) and 3 other proteins, TTG2 (WRKY), GL2 (Homeobox-leucine zipper) and TOZ (WD40). To get insights into the multi-interaction of MiTTG1 with other proteins, the BiFC assay was employed to detect physical interaction between MiTTG1 and 3 proteins (MiMYBO, MiTT8 and MibHLH1). The BiFC results showed that GFP was only detected when MiTTG1 was co-expressed with MiMYBO, MiTT8 and MibHLH1, respectively while no GFP was captured when MiTTG1 was replaced by a free vector (Fig. 2C). The BiFC assay demonstrated the interaction between MiTTG1 
A

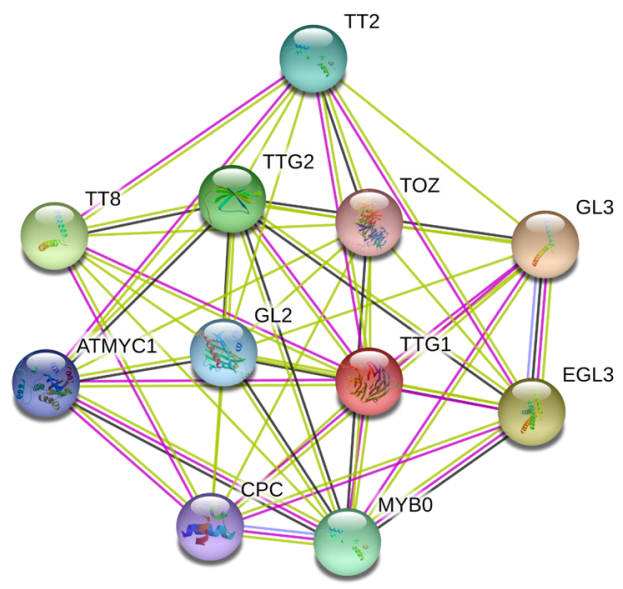

B
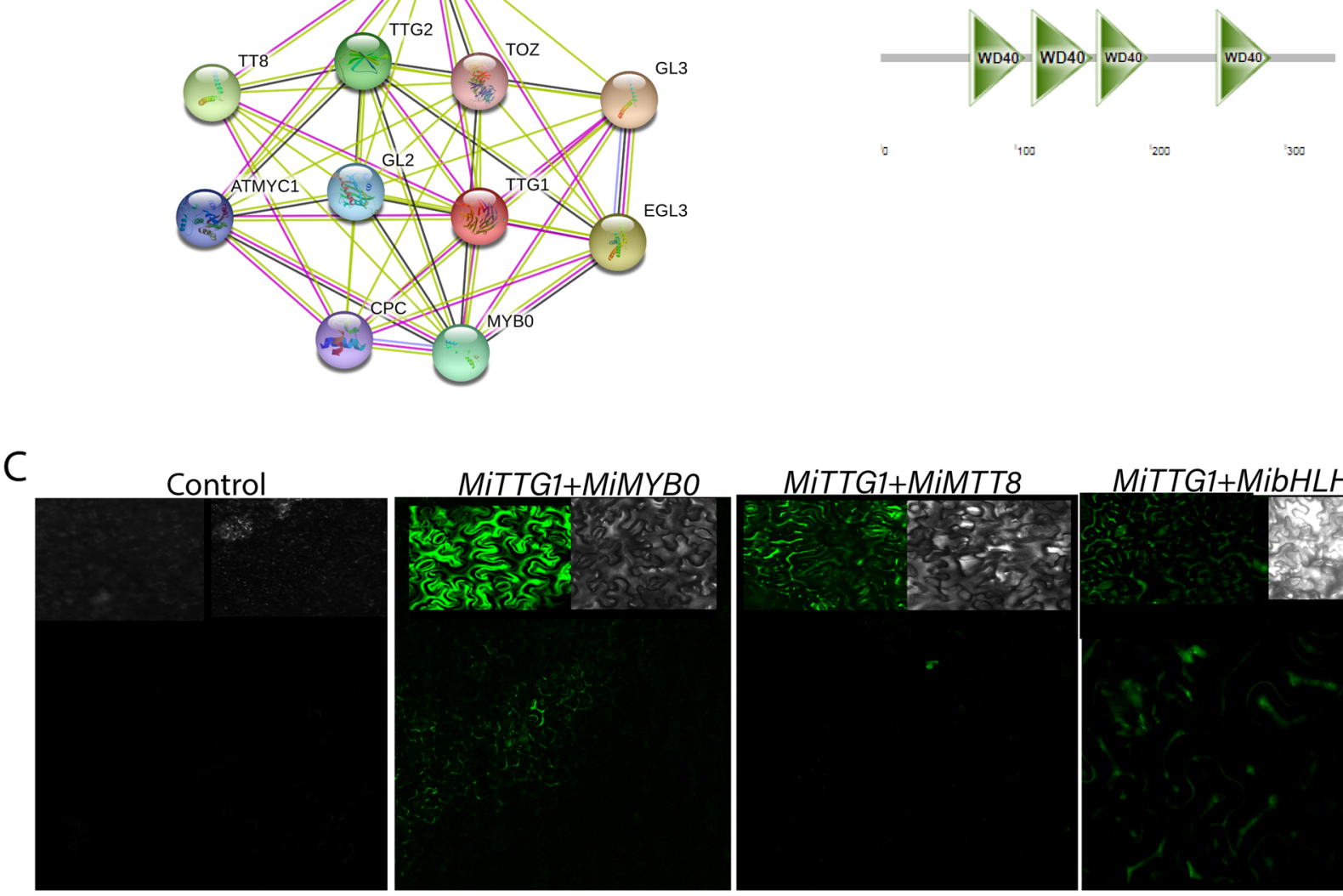

MiTTG1+MibHLH1

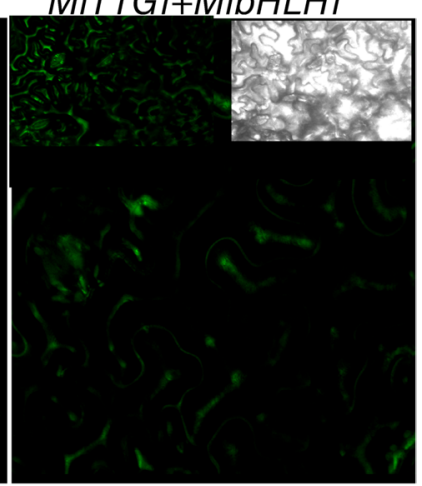

Figure 2. Protein-protein interaction networks of MangoWD40 proteins. (A) The prediction of protein interaction networks of WD40 with other transcription factors by STRING database. Each node is represented a protein and each edge is represented an interaction between two proteins. (B) Four conserved motifs of MiTTG1 protein. (C) MiTTG1 physically interacts with MiMYB, MiTT8 and MibHLH transcription factors in tobacco leaves were observed by confocal microscopy.

with MiMYBO, MiTT8 and MibHLH1 in tobacco leaf cells, implying that a new ternary regulatory mango MYBbHLH-WD40 might be functioned and shaped in a transgenic plant. The study result is consistent with earlier findings of the interaction of WD40-MYB-bHLH proteins in plants ${ }^{10,11,27,40}$.

MiTTG1 is involved in the regulation of root growth and development. Root hair systems that root epidermal cells are tubular-shaped and are important for nutrient acquirement, environmental interactions and soil anchorage in higher plants ${ }^{41}$. To elucidate the function of the MiTTG1 in root development, we systematically investigated the root phenotypes in young Arabidopsis transgenic lines (MiTTG1 was introduced into the wild-type) and wild-type. The transgenic Arabidopsis lines containing MiTTG1 were generated and confirmed by RT-PCR (Fig. 3A). The transgenic lines had higher root hair density than the wild-type plants (Fig. 3B). For 21 day-old plants, the relative root hair density and root lengths were higher and longer in transgenic lines as compared to the wild type (Fig. 3C). Taken together with the above data, this indicates that MiTTG1 enhances root hair density and root length, which suggests that the increased root system in the MiTTG1 transgenic line is promoted by its involvement in root growth and development. In Arabidopsis thaliana, AtTTG1 plays opposite roles in the regulation of root hairs and leaf trichome differentiation ${ }^{33,37}$. The heterologous expression of LbTTG1 in Arabidopsis plants decreased root hair density and increased trichome numbers ${ }^{42}$. The interaction between genetic factors and environmental signals is a key player for the determination of both the length and the abundance of root hairs ${ }^{43}$.

MiTTG1 overexpression confers tolerance to mannitol and salt stress during the early stages of root development in Arabidopsis. To further examine whether MiTTG1 is involved in abiotic stress responses in plants, we conducted a reverse genetic method to generate transgenic Arabidopsis lines from wildtype (overexpressing MiTTG1). The T3 homozygote lines of transgenic Arabidopsis had a single copy of MiTTG1 (Fig. 3A) and were selected for seed germination rate and root phenotypic analysis. The result showed that there was no significant difference in seed germination stage between the transgenic lines and the wild-type under normal conditions on $1 / 2$ MS (Fig. 4A). In the presence of mannitol and salt, the germination rate of both MiTTGloverexpression in transgenic lines and wild type seed was relatively reduced, but the reduction of wild type 
A
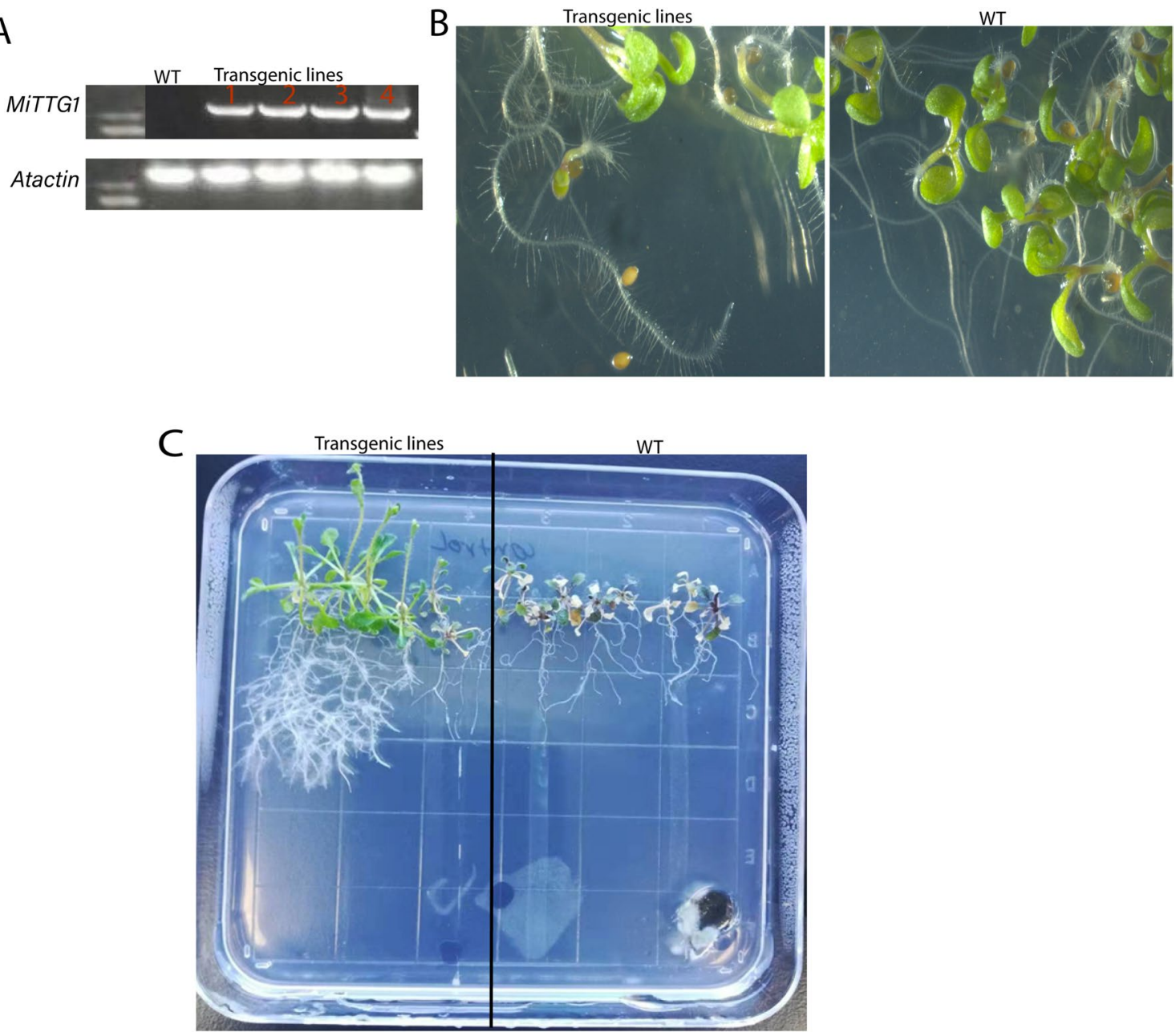

Figure 3. Expression pattern of MiTTG1 in Arabidopsis. The transcripts expression levels of the inserted MiTTG1 of T1 transgenic Arabidopsis lines confirmed by RT-qPCR with biological replicates. (A) Phenotypes of root and root hairs of wild-type and transgenic lines after 7 days post germination on $1 / 2 \mathrm{MS}$. (B) Phenotypes of root and root hairs of wild-type and transgenic lines after 21 and 30 days post germination on $1 / 2 \mathrm{MS}$.

was greater than that of transgenic seedlings. Under the treatment of $300 \mathrm{mMmannitol}$, about $89 \%$ of the transgenic seeds germinated, while only $67 \%$ of wild type seeds germinated at 5 days. Remarkably, $75.3 \%$ of the transgenic seeds germinated on $1 / 2 \mathrm{MS}$ supplemented with $200 \mathrm{mMNaCl}$, while only $43.3 \%$ of the wild-type seeds germinated after 5 days of treatment (Fig. 4A and Supplementary Table 3). MiTTG1 overexpression enhanced the tolerance of salt and mannitol within Arabidopsis transgenic lines, indicating that WD40 proteins may play a role in coordinating the plant molecular responses to environmental stresses. To analyze the effect of the mannitol and salt treatments on root growth, MiTTG1 overexpression lines and the wild-type were used against different concentrations of mannitol and salt. The result showed that the root lengths differed between transgenic lines and wild-type plants under the mannitol and salt treatments at 21 days (Fig. 4B,C and Supplementary Table 3). The transgenic lines showed higher root hair density and longer root lengths than in wild type under various concentrations of mannitol treatments (Fig. 4D and Supplementary Fig. 3), demonstrating increased tolerance to osmotic stress. The root lengths significantly increased in transgenic lines by $24 \%, 20 \%$ and $22 \%$ with various concentrations of mannitol, 100, 200 and $300 \mathrm{mM}$, respectively. Under salt treatment 50, 100, 150, $200 \mathrm{mM}$, the level root lengths increased by $15 \%, 10 \%, 10 \%$ and $4 \%$, respectively in transgenic lines compared with the wild-type as shown in Supplementary Table 3. The finding of these results demonstrates that MiTTG1 could play a vital role in plant responses to salt and osmotic tolerance during germination and post-germination stages. Overexpression of LbTTG1 increased the adaptability of transgenic Arabidopsis lines to NaCl treatment ${ }^{42}$.

MiTTG1 overexpression confers drought and stress-related marker genes. In order to understand the possible biological function of the MiTTG1 gene in the drought stress tolerance, transgenic lines and wild-type plants were exposed to drought situations by withholding water for 14 days (Fig. 5). It was observed that all the transgenic lines recovered while some wild type plants died after rewatering plants for 4 days (Fig. 5A). In general, wild type plants showed a more sensitive phenotype with chlorosis and more wilting of rosette leaves than the transgenic lines (Fig. 5A). It shows that MiTTG1 might be essential for mechanism-regulated plant stomatal closure in Arabidopsis plants in response to drought stress. To investigate the mechanism by 
A

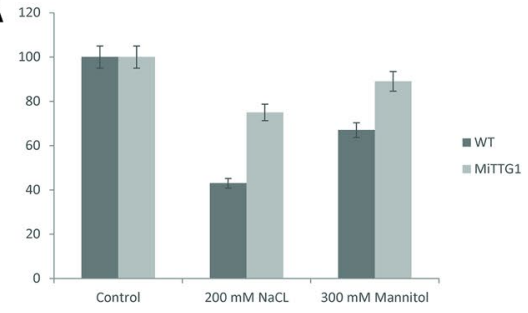

B

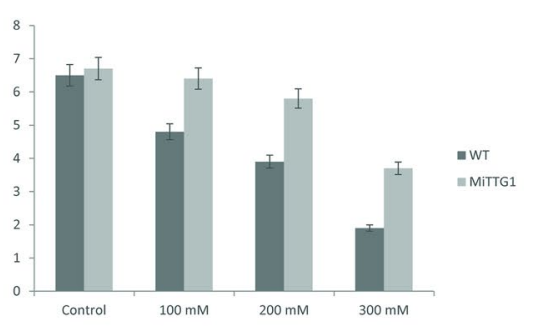

C

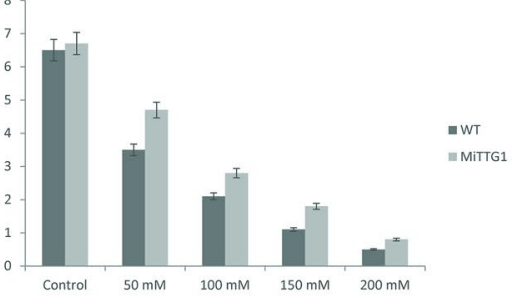

D

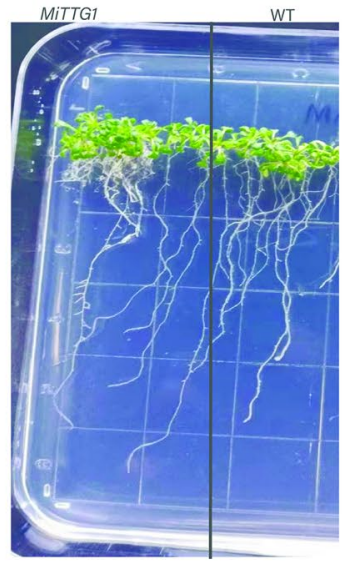

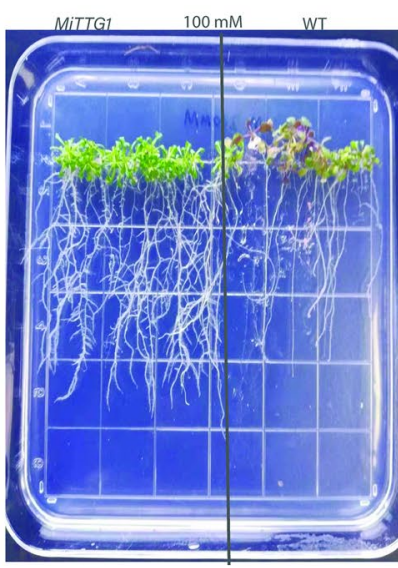
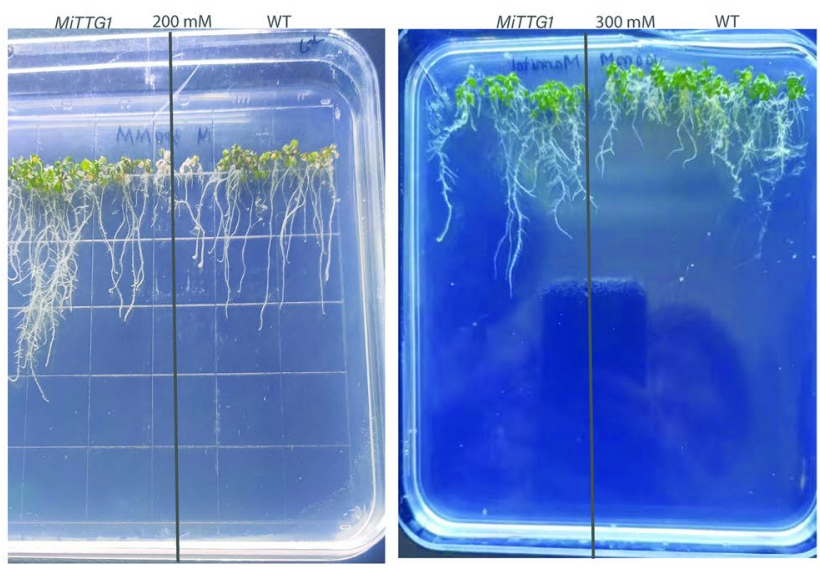

Figure 4. Overexpression of MiTTG1 enhances abiotic stress tolerance. (A) Seed germination rate of transgenic lines and wild type on $1 / 2$ MS supplemented with $300 \mathrm{mM}$ of mannitol and $200 \mathrm{mM}$ for 5 days. (B) Root length comparisons of transgenic lines and wild type on $1 / 2 \mathrm{MS}$ supplemented with different concentrations of mannitol (control, 100, 200, and $300 \mathrm{mM}$ ) for 21 days. (C) Root length comparisons of transgenic lines and wild type on $1 / 2$ MS supplemented with various concentrations of salt (control, 50, 100, 150, and $200 \mathrm{mM}$ ) for 21 days. (D) Root elongation and root density comparisons of transgenic lines and wild type on $1 / 2$ MS complemented with mannitol (control, 100, 200, and $300 \mathrm{mM}$ ) for 21 days.

which MiTTGloverexpression affects the drought tolerance phenotype of transgenic lines and wild type plants, we compared the expression of known stress-related genes, such as WDR5a, KIN1, KIN3 and PDF2 (Fig. 5B). These stress-related genes have been extensively examined in relation to drought stress tolerance in Arabidopsis $^{12,44}$. Drought-stress experiment showed that the expression levels of all stress-related genes and MiTTG1 were highly prompted in the transgenic lines by drought stress as compared to wild type (Fig. 5B), suggesting that overexpression of MiTTG1 gene has a positive role in enhanced drought stress resistance in these plants. Our findings showed that for the first-time, MiTTG1 plays a functional role in regulating abiotic stress tolerance in Arabidopsis. In other plants, TTG1 gene controls various features of plant growth and development such as proanthocyanidin and anthocyanin, plant defense $e^{45}$, root growth, seed coat pigment accumulation and leaf trichome differentiation ${ }^{33,37}$.

\section{Conclusion}

The WD40 protein family has various copies of the WD40 domain which fold into $\beta$-propeller arrangement thus acts as a scaffold for many protein-protein interactions. A total of 315 WD40 proteins were identified in mango genome and divided into 11 subgroups based on phylogenetic analysis. The biological and molecular functional grouping indicated that the mangoWD40 proteins are associating with many cellular functions in higher plants. WD40 protein sequences are mainly localized in the nucleus and cytoplasm while the remaining distributed in other subcellular membranes. The BiFC assay showed that MiTTG1 physically interacts with MiMYB0, MiTT8 and MibHLH1 in the tobacco leaf, suggesting that a new ternary regulatory mango MYB-bHLH-WD40 might be formed in transgenic plants. The findings indicate that MiTTG1 confers Arabidopsis improved tolerance to abiotic stress by regulating root growth and development. This first-time analysis of WD40 proteins in mango tropical fruit will provide important evidence of the functional variations among the mango tropical fruit.

\section{Methods}

Identification of mango WD40 proteins. Mango (Mangifera indica) genome assembly and genome annotation sequences were obtained from NCBI-BioProject (https://www.ncbi.nlm.nih.gov/bioproject/PRJNA $487154)^{25}$ for further comprehensive analysis. The HMM (Hidden Markov Model) folder of the WD40 protein domain (PF00400.31) was obtained from the Pfam (http://pfam.xfam.org/) database ${ }^{46}$ and was used as a query to search against the mango genome using HMMER software (version 3.1b2; http://hmmer.org/) with a default E-value $<10^{-10}$. The consistent protein sequences from Arabidopsis, peach and cotton species were obtained from the TAIR database (TAIR; http://www.Arabidopsis.org/), Phytozome database (https://phytozome.jgi.doe. 


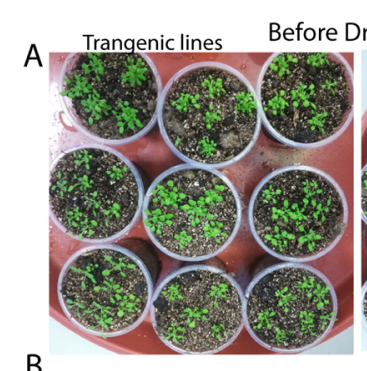

B
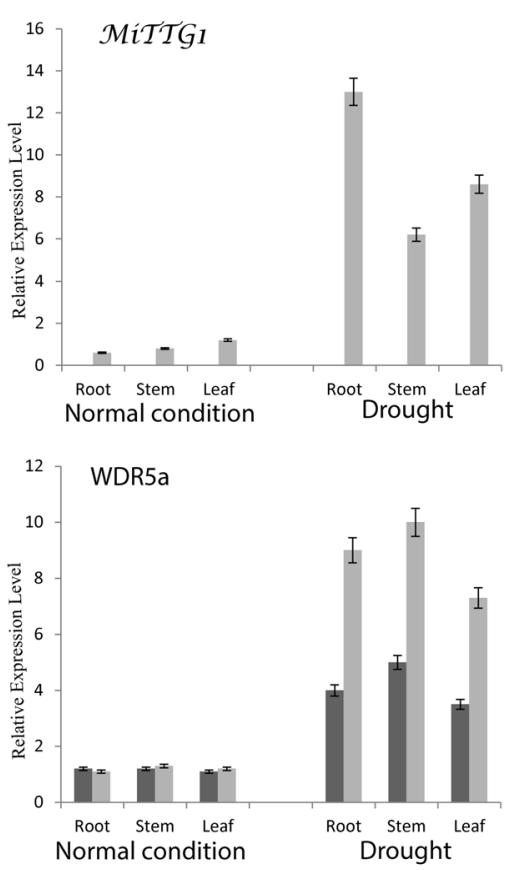
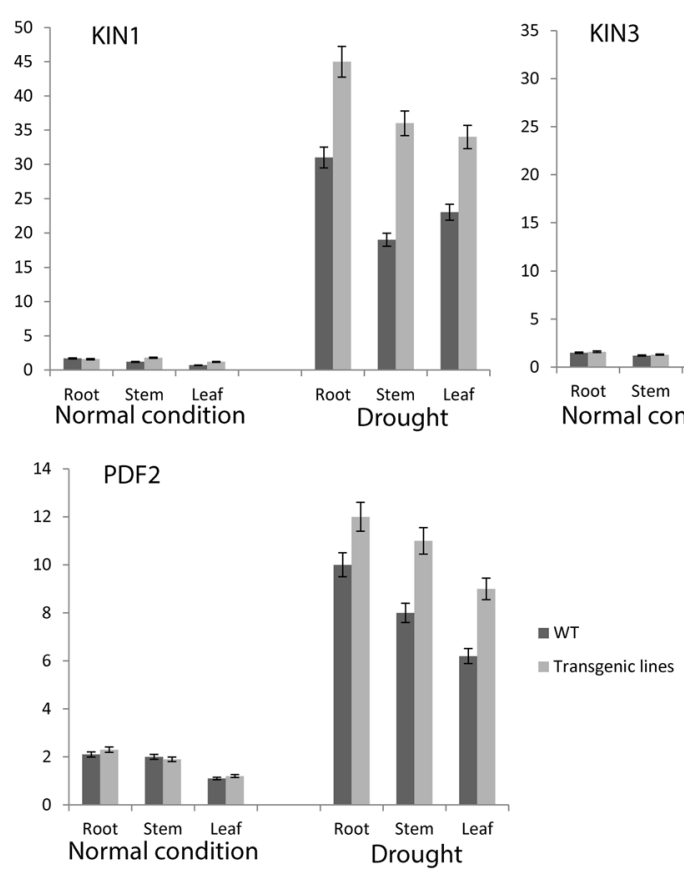
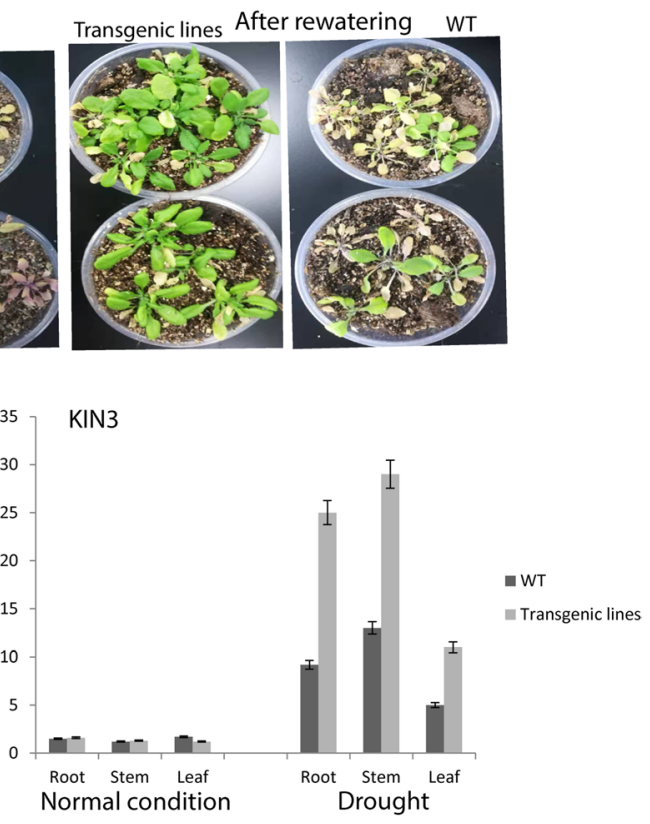

m Transgenic lines

Figure 5. Overexpression of MiTTG1 enhances drought stress resistance. (A) Drought stress treatments of transgenic lines and wild-type. 3 week old plants in soil were subjected to drought stress by withholding water for 14 days and the Arabidopsis plants were photographed after rewatering for 4 days. (B) Expression levels of MiTTG1 and stress-related genes (WDR5a, KIN1, KIN3 and PDF2) in transgenic lines and wild type. At actin was used as the gene reference, each experiment was done three times.

gov/) and cottongen website (https://www.cottongen.org), respectively and also were used as a query to search against mango genome database using local blast tool with a default E-value $<10^{-10}$. The Perl program was used to remove the redundant sequences among the identified gene sequences. The SMART database (http://smart .embl-heidelberg.de/) and the Pfam database (http://pfam.sanger.ac.uk/) were also used to ensure all mango WD40 protein sequences contained the WD40 domain. Only the mango protein sequences with WD40 domain were used for further investigations. Furthermore, the ExPASy Server tool (http://web.expasy.org/compute_pi/) was applied to predict the molecular weights and of isoelectric points WD40 proteins. The WoLFPSORT database (http://wolfpsort.org/) was also used to examine the subcellular localization of MiWD40 proteins. The chromosomal positions of WD40 members were done through blastN queries against the mango genome. MapChart software was used to generate a physical map of MiWD40 genes.

Phylogenetic analysis and conserved motif distribution and Gene Ontology (GO) Annotation of WD40 proteins in mango. The phylogenetic analysis of WD40 protein sequences was performed using multiple sequence alignments of mango and Arabidopsis WD40 protein members with ClustalW (http://www. ebi.ac.uk/Tools/msa/clustalw2/). MEGA 6.0 software (http://www.megasoftware.net) was used to generate an unrooted phylogenetic tree analysis using N-J ((Neighbor-Joining)) method with a bootstrap of 1000 replicates, p-distance and pairwise deletion. To predict the features of homologous WD40 domain and the occurrence of the common amino acids detected at each location in every repeat of the MiWD 40 protein domains, we searched common motifs shared by WD40 protein sequences by uploading their sequences to the online tool Multiple Expectation Maximization for Motif Elucidation (MEME) system (Version 4.9.1, http://meme.nbcr. net $/ \mathrm{meme} /)^{47}$. The parameters were set as follows: any number of repetitions, the optimum width from 6 to 250 and the maximum number of conserved motifs-10. The functional analysis of MiWD40 protein sequences was conducted by OmicsBox/Blast2GO (https://www.blast2go.com/) based on their biological process, cellular components and molecular functions. The amino acid sequence of every MiWD40 protein was incorporated by way of a fast format in the OmicsBox/Blast2Go online tool and blastp against protein sequence of NCBI, anno- 
tation and mapping to examine a protein function was done. The WD40 proteins were analyzed by Interpro in OmicsBox/Blast2Go and GO terms were combined with the annotated sequence mentioned previously. To gain more insight into the regulatory role of MiWD40 proteins in biological function in protein-protein interaction, the STRING online database (https://string-db.org/) ${ }^{48}$ was used to conduct the protein interaction networks of TTG1 protein.

Plant materials. The seeds of Arabidopsis thaliana (wild-type, Col-0) used in this work were obtained from Arabidopsis Biological Research Centre (ABRC). These seeds were planted at $22{ }^{\circ} \mathrm{C}$ in a growth chamber with a 16-h/8-h light/dark cycle. 5-day-old seedlings grown on half-strength Murashige and Skoog medium (1/2 MS) plates were transplanted into the $1 / 2 \mathrm{MS}$ and soil in a growth chamber at $22{ }^{\circ} \mathrm{C}$ in a $16-\mathrm{h} / 8$-h light/dark cycle. Tobacco plants were also used in this study for protein-protein interaction networks. Tobacco seeds were grown into the soil in a growth chamber at $22^{\circ} \mathrm{C}$ under a $16-\mathrm{h}-/ 8-\mathrm{h}$ light/dark cycle. Five-week-old plants were infiltrated using Agrobacterium tumefaciens strain GV3101.

Vector construction and transformation of Arabidopsis thaliana. The mango TTG1 ORF was inserted into NC frame of pCAMBIA1304-vector by nimble cloning system described by Dr Yan et al. ${ }^{49}$ under the control of the CaMV 35S promoter (p35S:: MiTTG1) using primers pNC-TTG1-UF and pNC-TTG1-UR (Supplementary Table 2A) according to the nimble cloning system (Supplementary Table 2B). The p35S::MiTTG1 vector was introduced into Agrobacterium tumefaciens strain GV3101 and plated on LB agar supplemented with rifampicin $(50 \mu \mathrm{g} / \mathrm{mL})$ and kanamycin $(50 \mu \mathrm{g} / \mathrm{mL})$. A single kanamycin-resistant colony was isolated and used to start a 250 -mL culture in LB liquid media supplemented with rifampicin $(50 \mu \mathrm{g} / \mathrm{mL})$ and kanamycin $(50 \mu \mathrm{g} /$ $\mathrm{mL}$ ), which was incubated for 2 days at $28^{\circ} \mathrm{C}$ with checking at $200 \mathrm{rpm}$ until OD (optical density) of $1.0-1.5$ at $600 \mathrm{~nm}$ (OD600). Bacterial cells were pelleted by centrifugation at $5000 \mathrm{rpm}$ for $30 \mathrm{~min}$ and the supernatant discarded. The bacterial pellet was resuspended in $200 \mathrm{~mL}$ of $5 \%$ sucrose with $0.05 \%$ Silwet L77). Arabidopsis wild type (Col-0) was used for transformation, which was conducted using the floral dip method described by Clough and Bent ${ }^{50}$. T0 seeds were harvested upon the maturation stage and transgenic lines (T1) were identified by selection on $1 / 2$ MS plates supplemented with Hygromycin $(25 \mu \mathrm{g} / \mathrm{mL})$ and RT-PCR. The homozygous T3 generation was used for further genetic analyses.

Bimolecular fluorescence complementation (BiFC) assay. Wild type Nicotiana benthamiana was used to investigate transient expression following an agro-infiltration procedure that was done according to a published method ${ }^{49}$. The full-length CDS of mango MiTTG1, MiMYB0, MiTT8 and MibHLH1 genes were cloned into the pNC-BiFC vector that contained of two NC frames with various adapters. Four genes of interest flanked by the adapters were recombined into the vector by the Nimble Cloning System. The genes encoding MiTTG1, MiMYB0, MiTT8 and MibHLH1 were amplified with nimble cloning primers containing the adapters in NC frames (Supplementary Table 2A). The PCR products were introduced into the pNC-BiFC vector by Nimble Cloning System. The empty vectors and the recombinant plasmids were transformed into A. tumefaciens GV3101 using the freeze-thaw method and were transiently expressed in tobacco leaves by agro-infiltration. Two days after infiltration, the green fluorescent protein (GFP) signals were examined with a FluoView FV1000 confocal microscope (Olympus, Japan) in the transfected cells.

Abiotic stress analysis of transgenic plants. Arabidopsis wild-type (col-0) and homozygous T3 generation transgenic lines were used in abiotic stress experiments. For germination rate, $300 \mathrm{mM} / \mathrm{L}$ of mannitol and $200 \mathrm{mM} / \mathrm{L}$ salt were used to examine the effect abiotic stress on transgenic lines and wild type seeds. For each replicate 100 seeds were used to investigate the effect. For stress treatment, 3-day-old sterilized seedlings were transferred into $1 / 2 \mathrm{MS}$ supplemented with mannitol $(0,100,200$ and $300 \mathrm{mM} / \mathrm{L})$ and $\mathrm{NaCL}(0,50,100,150$ and $200 \mathrm{mM} / \mathrm{L})$. Three weeks later, root phenotypic traits were analyzed. Drought treatment was also done to 3 -week-old seedlings in soil with sufficient water by withholding watering for 14 days. Samples from transgenic lines and wild-type were collected from root, stem and leaves. Three biological replicates were conducted to guarantee results reliability. Experimental datasets were presented in the form of the mean of three values with the standard deviation \pm SD. The analysis for significance was performed using Student's t-test.

Plant materials, RNA isolation and qRT-PCR analysis. To detect the MiTTG1 overexpression, Arabidopsis samples were collected from transgenic lines and wild-type (col-0) from root, stem and leaves. RNA was extracted from Arabidopsis root, stem and leaves using the Tiangen (RNA Aprep Pure Plant Kit). The concentration and quality of RNA samples were tested using a NanoDrop 2000 spectrophotometer and gel electrophoresis. RNA samples with high quality were treated with DNase I (TaKaRa, Japan) to remove genomic DNA contamination. The cDNA was synthesized using the ReverTra Ace qPCR RT kit (TOYOBO, Japan) depending on the manufacturer's manual. qRT-PCR experiments were performed to measure the expression pattern of TTG1 and related marker genes (WDR5a, PIN1, PIN3 and PDF2) for drought. The qRT-PCR investigation was done using the Applied Biosystems 7500 Real-Time PCR-system and the SYBER premix ExTaq kit (TaKaRa. Japan). The amplification of the target gene was estimated by the SYBR Green fluorescence signal. The Arabidopsis constitutive $\beta$-actin was used as a reference gene and specific TTG1 and related marker genes primers were used for qRT-PCR. The primers used in this study were listed in Supplementary Table 2C. The following thermal cycle settings of qRT-PCR and the expression analysis of genes were performed according to Salih et al. ${ }^{15}$.

Data availability

All related data are available within the manuscript and its additional files. 
Received: 9 May 2020; Accepted: 12 January 2021

Published online: 26 January 2021

\section{References}

1. Stirnimann, C. U., Petsalaki, E., Russell, R. B. \& Müller, C. W. WD40 proteins propel cellular networks. Trends Biochem. Sci. 35, 565-574 (2010).

2. Mishra, A. K., Muthamilarasan, M., Khan, Y., Parida, S. K. \& Prasad, M. Genome-wide investigation and expression analyses of WD40 protein family in the model plant foxtail millet (Setaria italica L.). PLoS ONE 9, 1-13 (2014).

3. Zhang, C. \& Zhang, F. The multifunctions of WD40 proteins in genome integrity and cell cycle progression. J. Genom. 3, 40-50 (2015).

4. van Nocker, S. \& Ludwig, P. The WD-repeat protein superfamily in Arabidopsis: Conservation and divergence in structure and function. BMC Genom. 4, 50 (2003).

5. Neer, E. J., Schmidt, C. J., Nambudripad, R. \& Smith, T. F. The ancient regulatory-protein family of WD-repeat proteins. Nature 371, 297-300 (1994).

6. Smith, T. F. Diversity of WD-repeat proteins. Subcell. Biochem. 978, 387-09595 (2008).

7. Gachomo, E. W., Jimenez-Lopez, J. C., Baptiste, L. \& Kotchoni, S. O. GIGANTUS1 (GTS1), a member of Transducin/WD40 protein superfamily, controls seed germination, growth and biomass accumulation through ribosome-biogenesis protein interactions in Arabidopsis thaliana. BMC Plant Biol. 14, 37 (2014).

8. Ekman, D., Light, S., Björklund, Å. K. \& Elofsson, A. What properties characterize the hub proteins of the protein-protein interaction network of Saccharomyces cerevisiae?. Genome Biol. 7, 1-13 (2006).

9. Nakatsuka, T. et al. Identification and characterization of R2R3-MYB and bHLH transcription factors regulating anthocyanin biosynthesis in gentian flowers. Plant Cell Physiol. 49, 1818-1829 (2008).

10. Zhao, M., Morohashi, K., Hatlestad, G., Grotewold, E. \& Lloyd, A. The TTG1-bHLH-MYB complex controls trichome cell fate and patterning through direct targeting of regulatory loci. Development 135, 1991-1999 (2008).

11. Ramsay, N. A. \& Glover, B. J. MYB-bHLH-WD40 protein complex and the evolution of cellular diversity. Trends Plant Sci. 10, 63-70 (2005).

12. Liu, W. C. et al. WD40-REPEAT 5a functions in drought stress tolerance by regulating nitric oxide accumulation in Arabidopsis. Plant Cell Environ. 40, 543-552 (2017).

13. Xu, X. et al. Nucleocytoplasmic trafficking of the Arabidopsis WD40 repeat protein XIW1 regulates ABI5 stability and abscisic acid responses. Mol. Plant 12, 1598-1611 (2019).

14. Kong, D., Li, M., Dong, Z., Ji, H. \& Li, X. Identification of TaWD40D, a wheat WD40 repeat-containing protein that is associated with plant tolerance to abiotic stresses. Plant Cell Rep. 34, 395-410 (2014).

15. Salih, H., Gong, W., Mkulama, M. \& Du, X. Genome-wide characterization, identification, and expressionanalysis of the WD40 protein family in cotton. Genome 547, 539-547 (2018).

16. Ouyang, Y., Huang, X., Lu, Z. \& Yao, J. Genomic survey, expression profile and co-expression network analysis of OsWD40 family in rice. BMC Genom. 13, 100 (2012).

17. Hu, R. et al. Genome-wide identification and analysis of WD40 proteins in wheat (Triticum aestivum L.). BMC Genom. https:// doi.org/10.1186/s12864-018-5157-0 (2018).

18. Zou, X. D. et al. Genome-wide analysis of WD40 protein family in human. Sci. Rep. 6, 39262 (2016).

19. Feng, R. et al. Identification and characterization of WD40 superfamily genes in peach. Gene 710, 291-306 (2019).

20. Jahurul, M. H. A. et al. Mango (Mangifera indica L.) by-products and their valuable components: A review. Food Chem. 183, 173-180 (2015).

21. Yahia, E. M. Mango (Mangifera indica L.). In Postharvest Biology and Technology of Tropical and Subtropical Fruits: Cocona to Mango, vol. 4, 492-567 (2011).

22. Sivakumar, D., Jiang, Y. \& Yahia, E. M. Maintaining mango (Mangifera indica L.) fruit quality during the export chain. Food Res. Int 44, 1254-1263 (2011)

23. Ribeiro, S. M. R. \& Schieber, A. Bioactive compounds in mango (Mangifera indica L.). In Bioactive Foods in Promoting Health, vol. 34, 507-523 (2010).

24. Bally, I. S. E. Mangifera indica (mango), ver. 3.1. Species Profiles Pacific Isl. Agrofor. (2006).

25. Wang, P. et al. The genome evolution and domestication of tropical fruit mango. Genome Biol. 21, 60 (2020).

26. Humphries, J. A., Walker, A. R., Timmis, J. N. \& Orford, S. J. Two WD-repeat genes from cotton are functional homologues of the Arabidopsis thaliana TRANSPARENT TESTA GLABRA1 (TTG1) gene. Plant Mol. Biol. 57, 67-81 (2005).

27. Schaart, J. G. et al. Identification and characterization of MYB-bHLH-WD40 regulatory complexes controlling proanthocyanidin biosynthesis in strawberry (Fragaria $\times$ ananassa) fruits. New Phytol. 197, 454-467 (2013).

28. Ben-Simhon, Z. et al. A pomegranate (Punica granatum L.) WD40-repeat gene is a functional homologue of Arabidopsis TTG1 and is involved in the regulation of anthocyanin biosynthesis during pomegranate fruit development. Planta 234, 865-881 (2011).

29. Taheri, A., Jayasankar, S., Cline, J. A., Raizada, M. N. \& Pauls, P. K. A WD-repeat gene from peach (Prunus persica L.) is a functional ortholog of Arabidopsis thaliana TRANSPARENT TESTA GLABRA1. Vitr. Cell. Dev. Biol. Plant 48, 23-29 (2012).

30. Ciborowski, P. \& Silberring, J. Proteomic Profiling and Analytical Chemistry: The Crossroads: Second Edition. Proteomic Profiling and Analytical Chemistry: The Crossroads: Second Edition (2016). https://doi.org/10.1016/C2015-0-01616-1.

31. Li, Q. et al. Genome-wide analysis of the WD-repeat protein family in cucumber and Arabidopsis. Mol. Genet. Genom. 289, 103-124 (2014).

32. Zhu, Y. et al. Genome-wide identification, sequence characterization, and protein-protein interaction properties of DDB1 (damaged DNA binding protein-1)-binding WD40-repeat family members in Solanum lycopersicum. Planta 1, 1337-1350 (2015).

33. Ioannidi, E. et al. Trichome patterning control involves TTG1 interaction with SPL transcription factors. Plant Mol. Biol 98, 1-13 (2016).

34. Gonzalez, A. et al. TTG2 controls the developmental regulation of seed coat tannins in Arabidopsis by regulating vacuolar transport steps in the proanthocyanidin pathway. Dev. Biol. 419, 54-63 (2016).

35. Caro, E., Castellano, M. M. \& Gutierrez, C. A chromatin link that couples cell division to root epidermis patterning in Arabidopsis. Nature 447, 213-217 (2007).

36. Zhang, F., Gonzalez, A., Zhao, M., Payne, C. T. \& Lloyd, A. A network of redundant bHLH proteins functions in all TTG1dependent pathways of Arabidopsis. Development 130, 4859-4869 (2003).

37. Gan, L., Xia, K., Chen, J. G. \& Wang, S. Functional characterization of TRICHOMELESS2, a new single-repeat R3 MYB transcription factor in the regulation of trichome patterning in Arabidopsis. BMC Plant Biol. 71, 1815-1827 (2011).

38. Zimmermann, I. M., Heim, M. A., Weisshaar, B. \& Uhrig, J. F. Comprehensive identification of Arabidopsis thalianaMYB transcription factors interacting with R/B-like BHLH proteins. Plant J. 40, 22-34 (2004).

39. Dreze, M. et al. Evidence for network evolution in an Arabidopsis interactome map. Science 8, 601-607 (2011).

40. Balkunde, R., Pesch, M., Hu, M. \& Hülskamp, M. Trichome patterning in Arabidopsis thaliana: From genetic to molecular models. Curr. Top. Dev. Biol. 91, 299-321 (2010). 
41. Feng, Y. et al. Ethylene promotes root hair growth through coordinated EIN3/EIL1 and RHD6/RSL1 activity in Arabidopsis. Proc. Natl. Acad. Sci. U. S. A. 114, 13834-13839 (2017).

42. Yuan, F. et al. A WD40-repeat protein from the recretohalophyte limonium bicolor enhances trichome formation and salt tolerance in Arabidopsis. Front. Plant Sci. 10, 1456 (2019).

43. Brueggemann, J., Weisshaar, B. \& Sagasser, M. A WD40-repeat gene from Malus ?? domestica is a functional homologue of Arabidopsis thaliana TRANSPARENT TESTA GLABRA1. Plant Cell Rep. 29, 285-294 (2010).

44. Sakuma, Y. et al. Functional analysis of an Arabidopsis transcription factor, DREB2A, involved in drought-responsive gene expression. Plant Cell 18, 1292-1309 (2006).

45. Miller, J. C., Chezem, W. R. \& Clay, N. K. Ternary WD40 repeat-containing protein complexes: Evolution, composition and roles in plant immunity. Front. Plant Sci. 6, 1108 (2016).

46. Finn, R. D. et al. Pfam: The protein families database. Nucleic Acids Res. 42, D222-D230. https://doi.org/10.1093/nar/gkt1223 (2014).

47. Bailey, T. L. et al. MEME Suite: Tools for motif discovery and searching. Nucleic Acids Res. 37, 202-208 (2009).

48. Szklarczyk, D. et al. STRING v10: Protein-protein interaction networks, integrated over the tree of life. Nucleic Acids Res. 43, 447-452 (2015).

49. Yan, P. et al. Nimble cloning: A simple, versatile, and efficient system for standardized molecular cloning. Front. Bioeng. Biotechnol 7, 2296-4185 (2020).

50. Clough, S. J. \& Bent, A. F. Floral dip: A simplified method for Agrobacterium-mediated transformation of Arabidopsis thaliana. Plant J. 16, 735-743 (1998).

\section{Acknowledgements}

The authors appreciate the support of the Central Public-interest Scientific Institution Basal Research Fund for Chinese Academy of Tropical Agricultural Sciences, grant number (No1630092020012) and the earmarked fund for the Belt and Road Tropical Project (BARTP-07). We thank Dr. Pu Yan (Institute of Tropical Bioscience and Biotechnology, Chinese Academy of Tropical Agricultural Sciences, Haikou, China) for providing Tabaco seeds, nimble cloning vectors and technical advice.

\section{Author contributions}

H.S., L.T. and F.A. designed the experiment. H.S., N.N.W.H. and F.A. grew the seedlings and performed the experiments. H.S. analyzed the results and prepared the manuscript. L.T. and R.Z. and revised the manuscript. All authors reviewed and approved the final manuscript.

\section{Competing interests}

The authors declare no competing interests.

\section{Additional information}

Supplementary Information The online version contains supplementary material available at https://doi. org/10.1038/s41598-021-81969-z.

Correspondence and requests for materials should be addressed to H.S. or R.Z.

Reprints and permissions information is available at www.nature.com/reprints.

Publisher's note Springer Nature remains neutral with regard to jurisdictional claims in published maps and institutional affiliations.

(c) (i) Open Access This article is licensed under a Creative Commons Attribution 4.0 International License, which permits use, sharing, adaptation, distribution and reproduction in any medium or format, as long as you give appropriate credit to the original author(s) and the source, provide a link to the Creative Commons licence, and indicate if changes were made. The images or other third party material in this article are included in the article's Creative Commons licence, unless indicated otherwise in a credit line to the material. If material is not included in the article's Creative Commons licence and your intended use is not permitted by statutory regulation or exceeds the permitted use, you will need to obtain permission directly from the copyright holder. To view a copy of this licence, visit http://creativecommons.org/licenses/by/4.0/.

(C) The Author(s) 2021 